\title{
A Clinical Study on Pulsed Low Dose Rate Radiation Therapy for Recurrent Cancers
}

\author{
X Tong*, FS Luo, GR Yang, DP Wang, X Song, Y Chen, S Wu, N Zhang, QQ Xu, T Zhang, and J Li \\ $3^{\text {rd }}$ Affiliated Hospital of Qiqihar Medical University, Qiqihar, China
}

\section{ABSTRACT}

A consensus standard of care has not been established for treating recurrent cancer patients who have previously undergone radiation therapy (RT). Several in vitro and in vivo studies have shown that pulsed low-dose-rate RT (PLDR) has the potential to reduce normal tissue toxicities while still providing significant tumor control for recurrent cancers. This work investigated PLDR re-irradiation for patients with recurrent cancers in head and neck $(\mathrm{H} \& \mathrm{~N})$, lung and pelvis. Thirteen patients with recurrent cancer were treated with the PLDR technique between 2012 and 2014. The re-irradiation sites included neck nodes, lung, chest wall, thyroid, pelvic nodes, pelvic nodes, uterus, cervix and vagina, supraclavicular nodes, and spine. The previous dose was $\geq 50 \mathrm{~Gy}$ for all patients, while the re-irradiation dose was 16-60Gy. The interval between prior RT treatment and re-irradiation was 13 - 336 months, and the follow-up time was up to 27 months. The PLDR treatments were effective (CR: 3 patients, PR: 10 patients). The acute and late toxicities were all acceptable (generally grade 2 or under). Our preliminary clinical results demonstrated the effectiveness of the PLDR technique for the palliative treatment of recurrent H\&N, lung, rectal and gynecologic cancers. Further clinical trials are warranted to quantify the efficacy of PLDR for recurrent cancers and other radiation resistant cancers and/or large treatment volumes involving critical structures.

Keywords: Pulsed low dose-rate RT (PLDR), re-irradiation, recurrent cancer, dose rate effect, tumor control, normal tissue toxicities

\section{INTRODUCTION}

Many technological developments have been made for radiation therapy over the last several decades including computer controlled linear accelerators, multi-leaf collimators (MLC), treatment planning systems and optimization techniques, and various imaging modalities and treatment targeting/guidance techniques (Mayles et al. 2007). Radiobiology plays an important role in the design of new radiotherapy trials to explore novel treatment strategies such as dose escalation and hypo/hyper-fractionation that employ advanced delivery techniques such as intensity modulated radiation therapy (IMRT), volumetric modulated arc therapy (VMAT) and particle therapy (Kavanagh and Timmerman

\section{Vol No: 06, Issue: 01}

Received Date: December 06, 2021

Published Date: December 21, 2021

\section{*Corresponding Author}

\section{Xu Tong}

MD, $3^{\text {rd }}$ Affiliated Hospital of Qiqihar Medical University, Qiqihar, China

E-mail: cn_tongxu@163.com

Citation: Tong X. (2021). A Clinical Study on Pulsed Low Dose Rate Radiation Therapy for Recurrent Cancers. Mathews J Cancer Sci. 6(1):28.

Copyright: Tong X. (C) (2021). This is an open-access article distributed under the terms of the Creative Commons Attribution License, which permits unrestricted use, distribution, and reproduction in any medium, provided the original author and source are credited. 
2005, Ma and Lamax 2012, Favaudon et al. 2014, Ma 2019).

Pulsed low-dose-rate radiotherapy (PLDR) is a novel treatment technique designed for re-irradiation of recurrent cancers (Tomé and Howard 2007, Richards et al. 2009, Adkison et al. 2011, Ma et al. 2011). It takes advantages of the hyper-radiosensitivity (HRS) of tumor cells below a certain low dose level (i.e., the transition dose), which is generally greater than those of normal tissues, and the increased normal tissue repair at low dose rates (Joiner et al. 2001, Steel 2002, Short et al. 2001, Harney et al. 2004, Wykes et al. 2006, Marples and Collis 2008). This is achieved by dividing a daily radiotherapy treatment into a number of subfractions (pulses) with each subfractional dose less than the tumor transition dose but greater than the normal tissue transition dose so that the radiation damage repair is triggered in normal tissues but not in tumor cells, resulting in an improvement in the therapeutic ratio. PLDR treatment can be delivered on conventional clinical accelerators with simple 2D/3D conformal beam arrangements or on advanced beam delivery systems employing IMRT/VMAT and treatment optimization algorithms (Rong et al. 2011, Ma et al. 2012, Lin et al. 2013, Tyagi et al. 2013, Kang et al. 2014, Li et al. 2014, Murphy et al. 2017, Geurts 2017).

Extensive in vivo experiments have been carried out to test the hypothesis of PLDR in terms of tumor control probability (TCP) and normal tissue complication probability (NTCP). Longer tumor growth delay was observed in many human tumor xenografts treated with PLDR than with conventional RT (Park et al. 2011, Dilworth et al. 2013, Zhang et al. 2015, Wang et al. 2020). PLDR also improved normal tissue toxicities in treated animals with fewer normal neuronal cell deaths (Park et al. 2011), less brain damage (Lee et al. 2013), better preserved vasculature (Dilworth et al. 2013, Meyer et al. 2016), and less damage to the gastrointestinal system (e.g., the stomach) and the immune and hematopoietic systems (e.g., spleen and bone marrow) (Zhang et al. 2015). Several phase I clinical trials have been conducted to exploit the therapeutic potential of PLDR for cancer treatments (Richards et al. 2009, Ma and Meyer 2011, Adkison et al. 2011, Mohindra et al. 2013, Magnuson et al. 2014, Meyer 2017, Burr et al. 2020, Bovi et al. 2020, Meyer 2020). Preliminary results from these clinical trials and other pioneering studies have shown favorable outcomes in TCP and NTCP for various cancers especially for recurrent cancers, bulky/ radioresistant tumors and refractory patients (Richards et al. 2009, Adkison et al. 2011, Mohindra et al. 2013, Magnuson et al. 2014, Lee et al. 2018, Yan et al. 2018, Rogacki et al. 2018,
Witt et al. 2019, Burr et al. 2020, Chen 2020).

A consensus standard of care has not been established for treating recurrent cancer patients, who have previously undergone radiation. In this work, we explored the clinical potential of PLDR for recurrent cancers in head and neck $(\mathrm{H} \& \mathrm{~N})$, lung and pelvis. Thirteen recurrent patients were treated using the PLDR delivery technique with 3D conformal radiation therapy (3DCRT) and followed up for 27 months. Here we present the outcome of PLDR treatment including tumor control and acute/late normal tissue toxicities. Preliminary results of this study were presented at the American Society for Radiation Oncology (ASTRO) 2015 annual meeting (Tong et al. 2015).

\section{MATERIALS AND METHODS}

\section{The Patient}

Thirteen patients with recurrent cancer were recruited for this study. Among them, 10 were male and 3 were female, aged between 49 and 74 . The patients were treated with the PLDR technique between 2012 and 2014 using the 3DCRT treatment technique. The re-irradiation sites included neck nodes (3), lung (3), chest wall (2), thyroid (1), pelvic nodes (1), pelvic nodes, uterus, cervix and vagina (1), supraclavicular nodes (1), and spine (1). The interval between previous RT and re-irradiation was 13 - 336 months, and the follow-up time was up to 27 months.

\section{The PLDR technique}

PLDR is a novel treatment technique, which can be delivered using conventional clinical machines using both simple 2D/3D beam arrangements or advanced IMRT/VMAT treatment techniques. The hypothesis of PLDR is to take advantage of both the low-dose HRS for tumors and the increased DNA repair at low dose rates for normal tissues by dividing the total daily dose into subfractions (pulses) of small doses and delivering them within a limited time frame to achieve an effective low dose rate. In this work, we have followed the proposal of Tomé and Howard (2007) by dividing a daily dose of 2 Gy into 10 pulses of 0.2 Gy with a 3-minute time internal (from the start of one pulse to the start of the next), resulting in an effective dose rate of $0.067 \mathrm{~Gy} / \mathrm{minute}$.

\section{PLDR planning and treatment}

The patients were treated on a Siemens Primus linear accelerator (Siemens Medical Systems, Concord, CA) and the 3DCRT plans were created using the Topslane 3D TPS (Topslane Inc, Suzhou, China). The clinical target volume 
(CTV) for these patients ranged between 161 and 703cc. The previous dose was $>50$ Gy for all patients, while the re-irradiation dose ranged between 16 and 60 Gy. Optimal beam angles were used to avoid organs at risk (OAR) in order to meet the OAR dose constraints as much as possible, which were the same as those used in the initial treatment. The dose was prescribed to the centroid of the planning target volume (PTV). The maximum dose in the PTV was less than $110 \%$ of the prescription dose and the minimum dose was better than $98 \%$ of the prescription dose. The maximum dose per pulse at any point of the PTV was less than $0.3 \mathrm{~Gy}$, which is less than the transition dose for most tumors (Hall and Brenner 1991, Joiner et al. 2001, Wykes et al. 2006, Marples and Collis 2008). The daily 2 Gy dose was divided into 10 sub-fractions (pulses) of 0.2 Gy and delivered with a time interval of 3 minutes (using a stopwatch) to obtain an effective dose rate of $0.067 \mathrm{~Gy} /$ minute.

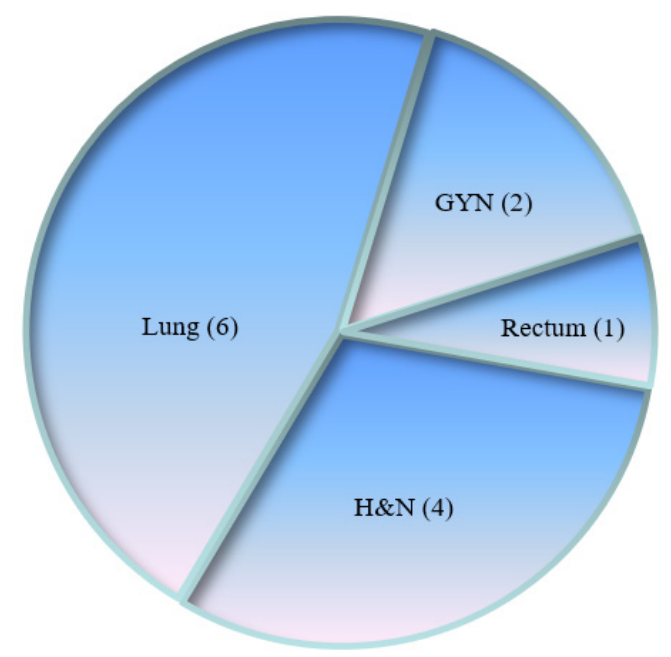

Figure 1: Number of patients per treatment site

\section{RESULTS}

We have treated 13 recurrent patients in this study. Primary cancer sites included head and neck, lung and pelvis (rectum and gynecologic cancers, GYN). Figure 1 shows the number of patients for different recurrent cancers. Table 1 shows the primary cancer sites, the previous doses received and the re-irradiation doses prescribed to the PLDR treatment volume in this study. The maximum prescription dose received in previous RT treatment was 66 Gy and the minimum prescription dose was $40 \mathrm{~Gy}$, which was for a previous stereotactic body radiotherapy (SBRT) patient. The maximum prescription dose for PLDR the re-irradiation treatment was 60 Gy and the minimum prescription dose was 16 Gy for a recurrent $H \& N$ patient.

Table 1: The previous dose received and the re-irradiation dose prescribed for the PLDR treatment.

\begin{tabular}{|c|c|c|}
\hline $\begin{array}{c}\text { Tumor } \\
\text { Site }\end{array}$ & Previous & Re-irradiation \\
\hline dose (Gy) & $66 \times 1$ & dose (Gy) \\
\hline H\&N & $60 \times 3$ & $60 \times 1$ \\
& & $50 \times 2$ \\
& & $16 \times 1$ \\
\hline
\end{tabular}




\begin{tabular}{|c|c|c|}
\hline & $60 \times 4$ & $60 \times 2$ \\
Lung & $54 \times 1$ & $50 \times 2$ \\
& $40($ SBRT $) \times 1$ & $48 \times 1$ \\
\hline GYN & & $30 \times 1$ \\
\hline Rectum & & $50 \times 1$ \\
\hline & $50 \times 2$ & $44 \times 1$ \\
\hline
\end{tabular}

The PLDR treatments were effective for all patients with good local control and acceptable normal tissue toxicities. Figure 2 shows MR images of a head and neck patient before and 10 weeks post PLDR treatment. Two patients died three months after the PLDR re-irradiation, one due to a massive cerebral infarction and the other due to acute cardiac failure.
All others survived more than 8 months by February 2015. Five patients showed good conditions at the last followup. Among them two recurrent lung cancer patients had survived 23 months and one nasopharyngeal cancer patient had survived 27 months.

Table 2: The clinical outcome for the 13 recurrent patients treated with PLDR.

\begin{tabular}{|c|c|c|c|}
\hline Tumor & Local & Acute toxicity & Late toxicity \\
& control & (grade) & Skin fibrosis $(3) \times 2$ \\
& & & Skin fibrosis $(2) \times 1$ \\
\hline H\&N & CR $\times 1$ & Xerostomia (1) $\times 2$ & Hyperpigmentation $(2) \times 3$ \\
& PR $\times 3$ & Mucositis (2) $\times 2$ & Xerostomia (1) $\times 3$ \\
\hline Lung & CR $\times 1$ & & Radiation pneumonitis (2) $\times 1$ \\
\hline GYN & PR $\times 5$ & & NO \\
\hline Rectum & PR $\times 2$ & GI toxicity (1) $\times 2$ & NO \\
\hline
\end{tabular}

Table 2 summarizes our clinical observations including local tumor control, acute and late normal tissue toxicities for the 13 patients treated with PLDR. Complete response (CR) was seen in 3 patients: 1 in H\&N, 1 in lung and 1 in rectum. Partial response (PR) was seen in 10 patients: 3 in H\&N, 5 in lung and 2 in GYN. The acute toxicities included xerostomia, mucositis, and GI toxicities, which were all grade 2 or under. The late toxicities included hyperpigmentation , xerostomia and radiation pneumonitis, which were all grade 2 or under, except for a $H \& N$ patient, who had grade 3 skin fibrosis. 


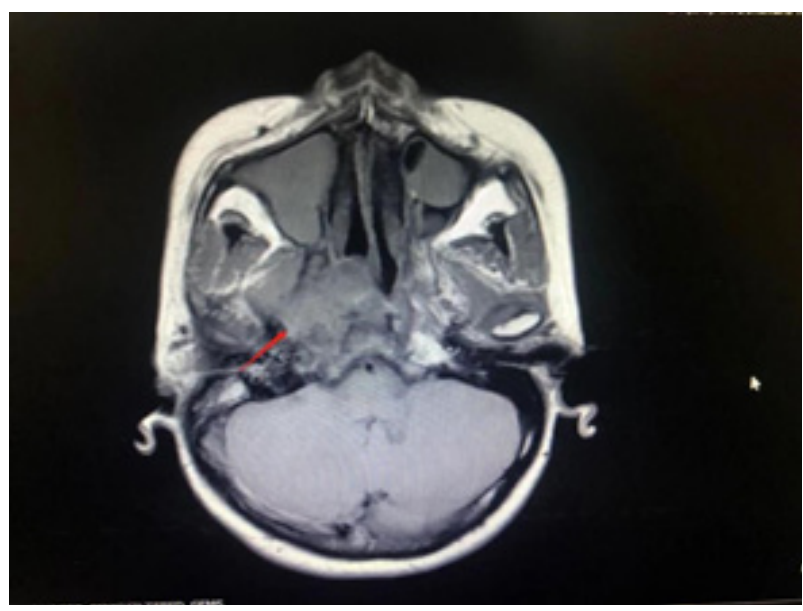

(a)

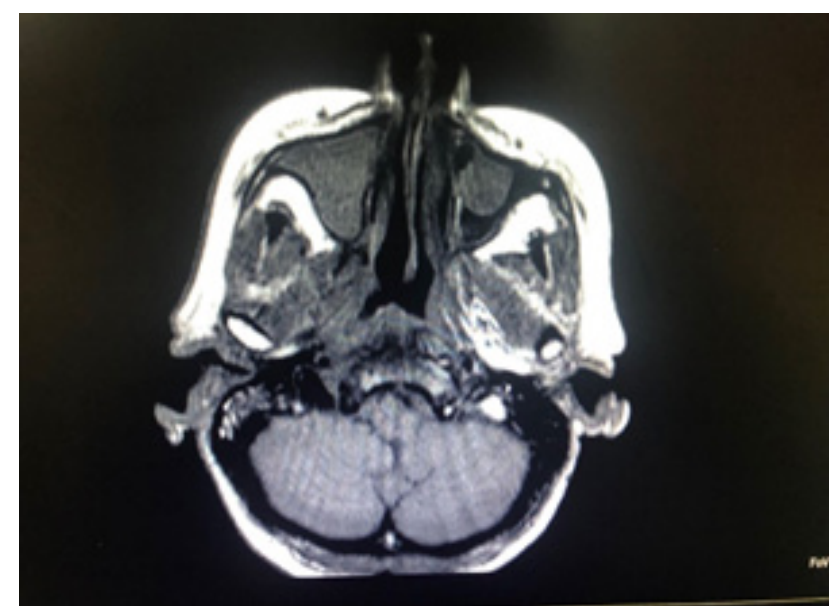

(b)

Figure 2: MR images of a nasopharyngeal patient showing favorable tumor control: (a) 1 week prior to and (b) 10 weeks post the PLDR treatment. The arrow in (a) points to the PLDR treated area.

\section{DISCUSSION}

PLDR has been explored by other investigators for treating recurrent $\mathrm{H} \& \mathrm{~N}$ cancer. A case report was presented by $\mathrm{Li}$ et al. (2012) on PLDR re-treatment of recurrent poorly differentiated squamous cell carcinoma of the nasopharynx metastatic to a cervical lymph node. The initial dose was 70 Gy to the gross tumor volume and a metastatic lymph node, and $\geq 50$ Gy to the bilateral cervical lymphatics. An additional 60 Gy was delivered to recurrent metastatic lymph nodes in the neck 5 years later. A second recurrence was discovered a year later and treated with PLDR of 70 Gy. In total, the patient received up to 190 Gy to the recurrence region. The recurrent lesion had a complete response to PLDR with no apparent radiation-induced normal tissue complications (only Grade 1 acute skin toxicity). In comparison, we treated $4 \mathrm{H} \& \mathrm{~N}$ recurrent patients using PLDR in this work. The total dose received by the recurrent tumor sites varied between 82 Gy and 126 Gy. We also achieved good local control (1 CR and 3 PR) with acceptable acute and late toxicities.

The Fox Chase group was the first to investigate advanced delivery techniques systematically for PLDR treatment of recurrent cancers to further reduce normal tissue toxicities (Ma et al. 2012, Lin et al. 2013, Li et al. 2014, Kang et al. 2014). These delivery techniques were used in their phase I dose escalation trial to investigate PLDR irradiation for palliation of recurrent tumors (Ma and Meyer 2011). Lee et al. (2019) evaluated 39 patients treated with PLDR reirradiation from 2009 to 2016 from their institution. The median follow-up time was 8.8 months and the median interval from the first radiation course and re-irradiation was 26.2 months. The median dose for the first and second course of radiation was 50.4 Gy and 50 Gy, respectively. The local progression rate was $16.5 \%$ at 6 months and $23.8 \%$ at 12 months. Of the 41 disease sites for the 39 patients, $25(61 \%)$ were in the thoracic region including non-small cell lung and esophagus. For these thoracic patients 12 developed grade 1, 4 developed grade 2 and 4 developed grade 3 acute toxicities, and only 1 patient developed grade 1 late toxicity. In our study, 6 patients were treated with 3DCRT for recurrent primary lung or metastases. The prescription dose from the previous treatment ranged from 40 to $60 \mathrm{~Gy}$ and the prescription dose for the PLDR retreatment ranged from 30 to 60 Gy (maximum dose in the overlapping region $120 \mathrm{~Gy}$ ). Good local tumor control was achieved with $1 \mathrm{CR}$ and 5 PR. No acute toxicities were observed and only one patient developed grade 2 radiation pneumonitis.

Paly et al. (2020) reported PLDR re-irradiation of 38 pelvic patients between 2010 and 2019 using 3DCRT, IMRT and VMAT delivery techniques. Median follow-up was 10.4 months. The tumor re-irradiation target site included prostate, rectal, bladder, gynecologic cancers, etc. The prior overlapping dose was 35 - 80 Gy EQD2 for 31 EBRT patients with known initial RT history. Four patients received initial LDR prostate brachytherapy (115 to $145 \mathrm{~Gy}$ ). Twenty-three patients were treated with definitive intent to 50 - 76 Gy. The one-year Kaplan-Meier local progression-free proportion based on clinical, biochemical, or radiographic response was $59 \%$ and 6 of 23 patients had no evidence of disease at their last follow-up. For 12 patients with available followup imaging, the best local tumor response was CR in 8\%, PR in $42 \%$, stable disease (SD) in $25 \%$, and progressive disease 
(PD) in 25\%. For 15 patients treated palliatively (28 to $60 \mathrm{~Gy}$ ), the one-year Kaplan-Meier local progression-free proportion based on clinical or radiographic response was $61 \%$ and the best local tumor response was PR in 33\%, SD in 53\%, and PD in 13\%. Acute skin/soft tissue grade 1 and 2 toxicity was $5.3 \%$ and $5.3 \%$, respectively. Late skin/soft tissue grade 2 toxicity was $2.6 \%$. Acute GU grade 1 and 2 toxicity was $7.9 \%$ and $21.1 \%$, respectively. Late GU grade 1,2 , and 3 toxicity was $13.2 \%, 10.5 \%$, and $5.3 \%$, respectively. Acute GI grade 1 and 2 toxicity was $7.9 \%$ and $7.9 \%$, respectively. Late GI grade 1,2 , and 3 toxicity was $13.2 \%, 5.3 \%$, and $2.6 \%$, respectively. In our study, 2 GYN and 1 rectal cancer patients were treated with PLDR with good outcome, the local tumor response was CR for the rectal patient and PR for the 2 GYN patients. Patients only had acute GI grade 1 toxicity without any late toxicities observed.

In conclusion, we have successfully treated 13 recurrent patients with PLDR. Our results showed favorable clinical outcome in tumor control and acute/late normal tissue toxicities as reported by other investigators. PLDR and infrequent concurrent chemotherapy provided tumor control for the majority of patients for whom few if any, other options for local treatment existed. PLDR represents a viable option in cancer management, especially for patients in whom re-irradiation is otherwise high risk.

\section{ACKNOWLEDGEMENT}

We would like to thank Dr. Charlie Ma and other colleagues at Fox Chase Cancer Center for useful discussions and clinical guidance on PLDR treatment and planning.

\section{REFERENCES}

1. Adkison JB, Tome W, Seo S, et al. (2011). Reirradiation of large-volume recurrent glioma with pulsed reduceddose-rate radiotherapy. Int J Radiat Oncol Biol Phys. 79:835-841.

2. Bovi JA, Prah MA, Retslaff AA, Schmainda KM, et al. (2020). Pulsed Reduced Dose Rate Radiotherapy in Conjunction with Bevacizumab or Bevacizumab Alone in Recurrent High-grade Glioma: Survival Outcomes, Int J Radiation Oncol Biol Phys. 108:979-986.

3. Burr AR, Robins HI, Bayliss RA and Howard SP. (2020). Pulsed Reduced Dose Rate for Reirradiation of Recurrent Breast Cancer, Practical Radiat Oncol. 10:e61-e70

4. Cannon GM, Tomé WA, Robins HI, Howard SP. (2007). Pulsed reduced dose-rate radiotherapy: case report: a novel re-treatment strategy in the management of recurrent glioblastoma multiforme. J Neurooncol 83:307-311.

5. Chen L. (2021). Clinical Applications of Pulsed Low DoseRate Radiation Therapy. Mathews J Cancer Sci. (6)1:22.

6. Dilworth JT, Krueger SA, Dabjan M, et al. (2013). Pulsed low-dose irradiation of orthotopic glioblastoma multiforme (GBM) in a pre-clinical model:effects on vascularization and tumor control. Radiother Oncol. 108:149-154

7. Favaudon V, Caplier L, Monceau V, Pouzoulet F, Sayarath M, Fouillade C, et al. (2014). Ultrahigh dose-rate FLASH irradiation increases the differential response between normal and tumor tissue in mice. Sci Transl Med. 6:245293.

8. Geurts M. (2017). TrueBeam Low Dose Rate Investigation for Pulsed Reduced Dose Rate IMRT. Int J Med Phys Clin Eng Radiat Oncol. 6:139-149.

9. Hall EJ, Brenner DJ. (1991). The dose-rate effect revisited: radiobiological considerations of importance in radiotherapy. Int J Radiat Oncol Biol Phys. 21:14031414.

10. Harney J, Short SC, Shah N, et al. (2004). Low dose hyperradiosensitivity in metastatic tumors". Int J Radiat Oncol Biol Phys. 59:1190-1195.

11. Joiner MC, Marples B, Lambin P, et al. (2001). Lowdose hypersensitivity: current status and possible mechanisms. Int J Radiat Oncol Biol Phys. 49:379-389.

12. Kang SW, Lang J, Wang P, Li J, Lin MH, et al. (2014). Optimization strategies for pulsed low-dose-rate IMRT of recurrent lung and head and neck cancers. J Appl Clin Med Phys. (15)3:102-113

13. Kavanagh BD, Timmerman RD. (2005). Stereotactic body radiation therapy, Lippincott Williams \& Wilkins, Philadelphia, PA. pp. 1-153.

14. Lee CT, Dong Y, Li T, Freedman S, Anaokar J, et al. (2018). Local control and toxicity of external beam reirradiation with a pulsed low-dose-rate technique. Int J Radiat Oncol Biol Phys. 100:959-964.

15. Li G-H, Zhu B, Yang F, Ma CK, Yang D-Q. (2012). Use of cetuximab in combination with pulsed reduced dose-rate radiotherapy in a patient with recurrence of nasopharyngeal carcinoma in the neck. Exp Ther Med. 3:869-872. 
16. Li J, Lang J, Wang P, Kang S, Lin MH, et al. (2014). Intensity-modulated radiation therapy for pancreatic and prostate cancer using pulsed low-dose rate deliver techniques. Med Dosimetry. 39:330-336, S0958-3947

17. Lin MH, Price RA, Li JS, Kang SW, Li J, et al. (2013). Investigation of pulsed IMRT and VMAT for re-irradiation treatments: dosimetric and delivery feasibilities. Phys Med Biol. 58:8179-8196

18. Luo F, Yang G, Zhang X, Li J, Gao J, et al. (2016). Clinical observation of reirradiation by pulsed low dose rate RT for recurrent cancer patients. J Qiqihar Medical Univ. 6:1153-1155

19. Ma C-M. (2019). Physics and Dosimetric Principles of SRS and SBRT. Mathews J Cancer Sci. 4(2):22, DOI: https://doi.org/10.30654/MJCS.10022.

20. Ma C-M and Lomax T. (2012). Ed., Proton and carbon ion therapy (Taylor \& Francis, New York).

21. Ma C-M and Meyer JE (Principal Investigators). (2011). Phase I Study of Pulsed Low Dose Rate Reirradiation Delivered with 3DCRT/IMRT for Palliation of Recurrent Tumors, Fox Chase Cancer Center, IRB\#11-044/OERRT-042. ClinicalTrials.gov Identifier: NCT01470365.

22. Ma C-M, Lin MH, Dai XF, et al. (2012). Investigation of pulsed low dose rate radiotherapy using dynamic arc delivery techniques. Phys Med Biol. 57:4613-4626.

23. Ma C-M, Lin MH, Kang SW, Li J, Wang P and Lang J. 2014 Pulsed Low-Dose-Rate Radiation Therapy (PLDR) for Recurrent Cancers: Treatment Planning Strategies for IMRT and VMAT, IJROBP 90:s941 DOI:https://doi. org/10.1016/j.ijrobp.2014.05.2659.

24. Ma C-M, Luxton G, Orton C. (2011). Point-Counter-Point: Pulsed reduced dose rate radiation therapy is likely to become the treatment modality of choice for recurrent cancers. Med Phys. 38:4909-4911.

25. Magnuson W, Robins HI, Mohindra P, Howard S. (2014). Large volume reirradiation as salvage therapy for glioblastoma after progression on bevacizumab. J Neurooncol. 117:133-139.

26. Marples B, Collis SJ. (2008). Low-dose hyperradiosensitivity:past, present, and future. Int J Radiat Oncol Biol Phys. 70:1310-1318.

27. Mayles P, Rosenwald JC, Nahum A. (2007). Handbook of Radiation therapy Physics: Theory and Practice. Taylor \& Francis. ISBN 978-0-7503-0860-1.
28. Meyer JE (Principal Investigator). (2017). Pulsed Low Dose Rate Radiation With Concurrent Chemotherapy for Non-Small Cell Lung Cancer and Esophageal Cancer, Fox Chase Cancer Center, IRB\# 16-1051/OER-RT-101. ClinicalTrials.gov Identifier: NCT03094884

29. Meyer JE (Principal Investigator). (2020). Utilizing Pulsed Low-Dose-Rate (PLDR) Radiation to Prevent de novo stromal Activation; a Neoadjuvant Pancreatic Adenocarcinoma Phase I Trial, Fox Chase Cancer Center, IRB\# 18-1085/OER-RT-155. ClinicalTrials.gov Identifier: NCT04452357.

30. Meyer K, Krueger SA, Kane JL, Wilson TG, et al. (2016). Pulsed Radiation Therapy With Concurrent Cisplatin Results in Superior Tumor Growth Delay in a Head and Neck Squamous Cell Carcinoma Murine Model. Int J Radiat Oncol Biol Phys. 96:161-9. doi:10.1016/j. ijrobp.2016.04.031.

31. Mohindra P, Robins IH, Tomé WA, Hayes L, Howard SP. (2013). Wide-field Pulsed Reduced Dose Rate Radiotherapy (PRDR) for Recurrent Ependymoma in Pediatric and Young Adult Patients. Anticancer Res. 33:2611-2618.

32. Murphy ES, Rogacki K, Godley A. (2017). Intensity modulated radiation therapy with pulsed reduced dose rate as a re-irradiation strategy for recurrent central nervous system tumors: An institutional series and literature review. Pract Radiation Oncol. 7:391-399.

33. Paly J, Deng M, Lee CT, Hayes SB, Galloway TJ, et al. (2020). Pelvic Reirradiation Utilizing Pulsed Low-dose Rate Radiation Therapy. Am J Clin Oncol. 43:748-751, doi:10.1097/COC.0000000000000741.

34. Park SS, Chunta JL, Robertson JM, Martinez AA, Oliver Wong CY, et al. (2011). MicroPET/CT imaging of an orthotopic model of human glioblastoma multiforme and evaluation of pulsed low-dose irradiation. Int J Radiat Oncol Biol Phys. 80:885-892.

35. Richards GM, Tome WA, Robins HI. (2009). Pulsed reduced dose-rate radiotherapy:a novel locoregional retreatment strategy for breast cancer recurrence in the previously irradiated chest wall, axilla, or supraclavicular region. Breast Cancer Res Treat. 114:307-313.

36. Rogacki K, Chao ST, Yu J, Godley A, Balagamwala E, et al. (2018). Review of Pulsed Reduced Dose Rate Reirradiation for Recurrent Tumors. J Cancer Clin Trials. 3:143. doi: 10.4172/2577-0535.10001 
37. Rong Y, Paliwal B, Howard SP and Welsh J. (2011). Treatment Planning for Pulsed Reduced Dose-Rate Radiotherapy in Helical Tomotherapy. Int J Radiat Oncol Biol Phys. 79:934-942.

38. Short SC, Woodcock M, Marples B, Joiner MC. (2003). Effects of cell cycle phase on low-dose hyperradiosensitivity. Int J Radiat Biol. 79:99-105.

39. Steel GG. (2002). Basic clinical radiobiology. 3rd ed. (Oxford Univ Press Inc., New York) pp. 192-204

40. Todorovic V, Prevc A, Zakelj MN, et al. (2020). Pulsed low dose-rate irradiation response in isogenic HNSCC cell lines with different radiosensitivity. Radiol Oncol. 54(2):168-179.

41. Tomé WA and Howard SP. (2007). On the possible increase in local tumour control probability for gliomas exhibiting low dose hyper-radiosensitivity using a pulsed schedule. Br J Radiol. 80:32-37.
42. Tong X, Luo F, Liu Y, Zhang W, Xu Q et al. (2015). Pulsed Low Dose Rate RT for Recurrent Cancers: A Clinical Observation. IJROBP. 93(3):E478 DOI:10.1016/j. ijrobp.2015.07.1767

43. Tyagi N, Yang K, Sandhu R, Yan D, Park SS, et al. (2013). External Beam Pulsed Low Dose Radiotherapy Using Volumetric Modulated Arc Therapy: Planning and Delivery. Med Phys. 40, Article Number: 011704.https:// doi.org/10.1118/1.4769119

44. Wang B, Ren J, Zhang Z, Cvetkovic D, Chen XM, et al. (2020). An In-Vivo Study on Pulsed Low-Dose-Rate Radiotherapy for Prostate Cancer. Mathews J Cancer Sci. 4(2):21 DOI:10.30654/mjcs.10021 